\title{
Resistance to Increasing Chemical Classes of Fungicides by Virtue of "Selection by Association" in Botrytis cinerea
}

\author{
Meng-Jun Hu, Kerik D. Cox, and Guido Schnabel
}

First and third authors: Department of Agricultural and Environmental Sciences, Clemson University, Clemson, SC; and second author: Department of Plant Pathology and Plant-Microbe Biology, Cornell University, Geneva, NY. Accepted for publication 3 August 2016.

\begin{abstract}
Hu, M.-J., Cox, K. D., and Schnabel, G. 2016. Resistance to Increasing Chemical Classes of Fungicides by Virtue of "Selection by Association" in Botrytis cinerea. Phytopathology 106:1513-1520.

Previous research has shown that Botrytis cinerea isolates with resistance to multiple chemical classes of fungicides exist in eastern strawberry fields. In this study, the fungicide resistance profiles of 2,130 isolates from flowers of commercial strawberry fields located in multiple states was determined over four consecutive strawberry production seasons. Producers were asked to alternate single-site fungicides that were considered low risk in their specific location based on resistance monitoring results in their fields. This recommendation led to an increase of chemical class diversity used in the spray programs. Results indicated that simultaneous resistance in

individual isolates to two, three, four, five, six, and seven classes of fungicides increased over time. The increase in chemical class resistances within isolates was likely due to a process we termed "selection by association", where fungicide resistance traits were often linked to the trait being selected rather than the selectable trait itself. Data analysis also indicated that the odds were highest for isolates resistant to one chemical class (1CCR) to be resistant to thiophanate-methyl; for 2CCR isolates to be resistant to thiophanate-methyl and pyraclostrobin; and for 3CCR isolates to be resistant to thiophanate-methyl, pyraclostrobin, and either cyprodinil or fenhexamid. We hypothesize that the more chemical classes are used in a spray program, the faster isolates will be selected with increasing numbers of chemical class resistances by virtue of selection by association if such isolates preexist in the population.
\end{abstract}

Fungicide resistance in fungal pathogen populations usually develops following the intensive use of fungicides for disease control. A combination of theoretical argument, experimental evidence, and practical experience all suggest that the development of fungicide resistance in populations of fungal pathogens is greatly favored by the sustained, sole use of fungicides with specific mechanisms of action (Brent and Hollomon 2007; van den Bosch and Gilligan 2008). Conversely, occasional fungicide use, interspersed by the use of other, unrelated chemical classes is viewed as unlikely to lead to resistance problems (Brent and Hollomon 2007). Many reviews have described strategies to meet the challenge of fungicide resistance (Brent and Hollomon 2007; Delp 1980; Ktiller and Scheinpflug 1987; Staub 1991; Staub and Sozzi 1984). Such fungicide resistance management strategies are designed to prevent or delay the development of fungicide resistance, including rotation and mixtures of fungicide from different chemical classes, restriction of the number of treatments applied per season, application only during emergence, and adherence to the manufacturer's recommended dose (Brent and Hollomon 2007).

Botrytis cinerea Pers., the causal agent of Botrytis fruit rot (BFR), is one of the most important pathogens of strawberry (Fragaria $\times$ ananassa) worldwide and one of the first plant pathogens to develop resistance to new classes of fungicides. Control of BFR in commercial strawberry fields largely relies on the application of fungicides during bloom and fruit ripening. In addition to the multisite fungicides captan and thiram, single-site fungicides of multiple chemical classes are currently available for BFR management in the

Corresponding author: G. Schnabel; E-mail address: schnabe@clemson.edu

*The $\boldsymbol{e}$-Xtra logo stands for "electronic extra" and indicates that two supplementary tables are published online.

http://dx.doi.org/10.1094/PHYTO-04-16-0161-R

(C) 2016 The American Phytopathological Society southeastern United States, including anilinopyrimidines (cyprodinil and pyrimethanil), methyl benzimidazole carbamates (MBC; thiophanatemethyl), dicarboximides (iprodione), hydroxyanilides (HA; fenhexamid), quinone outside inhibitors (QoI; pyraclostrobin), phenylpyrroles (fludioxonil), and succinate dehydrogenase inhibitors (SDHI; boscalid, fluopyram, fluxapyroxad, and penthiopyrad). However, resistance to several of these fungicide classes was previously found in $B$. cinerea populations from strawberry fields in the eastern United States (Fernández-Ortuño and Schnabel 2012; Fernández-Ortuño et al. 2012, 2013a,b; Grabke et al. 2013, 2014; $\mathrm{Li}$ et al. 2014b). In a recent study, individual $B$. cinerea isolates collected from Connecticut, Georgia, South Carolina, and Virginia with resistance to seven chemical classes were identified and characterized (Fernández-Ortuño et al. 2015). The study identified multiple resistances to thiophanate-methyl, iprodione, boscalid, pyraclostrobin, fenhexamid, and fludioxonil in isolates of $B$. cinerea. Resistance was based on target gene mutations, including E198A and F200Y in $\beta$-tubulin, I365 N/S in bos 1, H272R/Y in $s d h B$, G143A in cytb, T63I and F412S in erg27, and variations in transcription factor $m r r l$ leading to overexpression of $\operatorname{atr} B$ (Fernández-Ortuño et al. 2015).

Monitoring of resistance in fungal pathogens of agriculturally important crops has been done on a larger, area-wide scale to detect shifts in sensitivity to fungicides or to detect the prevalence of resistant strains in certain regions (Brent 1988). Although this has value for scientific studies and to fine-tune fungicide resistance management strategies, it does not recognize differences in sensitivity to fungicides in local pathogen populations, and is inappropriate for fungal pathogens with no means of long-distance $(>8 \mathrm{~km})$ dispersal. Strawberry fields, even when in close proximity, may have unique fungicide resistance profiles (Fernández-Ortuño et al. 2014). Given that multifungicide resistance is widespread in $B$. cinerea $(\mathrm{Li}$ et al. 2014a), it appears prudent that resistance management strategies such as mixtures of fungicides with different modes of action or rotations are designed and implemented based on location-specific resistance 
profiles. This provides individual growers with critical information for disease and resistance management (Schnabel et al. 2015).

Between the 2011-12 and 2014-15 strawberry seasons, our laboratory determined resistance profiles in strawberry fields in the eastern United States, and preliminary results from the first two seasons (2011-12 and 2012-13) were recently published (FernándezOrtuño et al. 2014). In that study, a considerable proportion of $B$. cinerea isolates collected during the 2012-13 season were resistant to thiophanate-methyl (85\%), pyraclostrobin $(59 \%)$, cyprodinil (17\%), and fenhexamid (26\%) (Fernández-Ortuño et al. 2014). Resistance profiling was continued and, over the last 4 years, 213 locations (strawberry fields) were investigated using 10 representative $B$. cinere $a$ isolates per location. Based on the fungicide resistance profile, resistance management recommendations were provided to the growers. If no resistance to a fungicide class was present in a field population, we recommended fungicide classes in rotation with one another throughout the season. If a low level of resistance to a fungicide class was detected in an individual field population ( 1 of 10 isolates; $10 \%$ ), we recommended to use that fungicide class only once during that season and in combination with captan or thiram, and to add and rotate fungicides of different chemical classes to the spray program. If a high level of resistance to a fungicide class was detected ( $\geq 2$ of 10 isolates; $\geq 20 \%$ ), it was recommended to not use that class that year and to add and rotate fungicides of different chemical classes to the spray program (Schnabel et al. 2015). A survey among participating growers conducted in 2014 revealed that all growers involved in the program had been following our recommendations over the last 4 years and all were satisfied with the level of disease control (Schnabel et al. 2015). However, it is not known how these resistance management programs, based on location-specific fungicide resistance profiles, affected the composition of resistance phenotypes in $B$. cinerea over time. The objectives of this study were to determine the long-term impact of fungicide resistance management programs on fungicide resistance profiles in $B$. cinerea populations from strawberry fields.

\section{MATERIALS AND METHODS}

Collection of $B$. cinerea isolates from strawberry fields. The majority of commercial strawberry plants in the southeastern United States are cultivated as an annual hill-cropping system, in which freshly dug bare-root plants or plugs provided by nurseries are planted in late summer to early fall for cropping in spring. Strawberry flowers are susceptible to $B$. cinerea infection and are the primary infection court for future fruit infections (Bristow et al. 1986). Between planting and beginning of bloom, growers may apply captan or thiram but typically do not spray selective fungicides. Then, between bloom and harvest, most growers may spray captan mixed or rotated with selective fungicides at label rates 12 to 15 times on weekly intervals. Between the 2011-12 and 2014-15 strawberry growing seasons, 2,130 B. cinerea isolates were collected from flowers of 213 fields in 11 states, including Alabama (1 field, 10 isolates), Arkansas (6 fields, 60 isolates), Connecticut (3 fields, 30 isolates), Georgia (5 fields, 50 isolates), Maryland (36 fields, 360 isolates), Missouri (1 field, 10 isolates), North Carolina (42 fields, 420 isolates), Ohio (1 field, 10 isolates), Pennsylvania ( 2 fields, 20 isolates), South Carolina (89 fields, 890 isolates), and Virginia (27 fields, 270 isolates) (Supplementary Table S1). Individual isolates were obtained by collecting 20 to 40 strawberry blossoms with degenerated, black tori and processing these as described previously (Fernández-Ortuño et al. 2014). From each collection, 10 isolates were collected from a single field. No more than two sets of 10 isolates per farm operation and per year were included in the study to ensure that no single operation was overrepresented. Results from fields in South Carolina are presented separately from other states because fields were consistently sampled over all experimental seasons, allowing for more precise monitoring of resistance profiles in the same fields over time.
Monitoring for fungicide resistance in $\boldsymbol{B}$. cinerea isolates from strawberry. Commercial formulations of cyprodinil (Vangard WG fungicide; Syngenta Crop Protection), iprodione (Rovral 4 Flowable; Bayer Crop Sciences), fenhexamid (Elevate 50 WDG; Arysta Life Science), thiophanate-methyl (Topsin M 70WP; United Phosphorus, Inc.), fludioxonil (Scholar SC fungicide; Syngenta Crop Protection), pyraclostrobin (Cabrio EG fungicide, 20\% wt/wt; BASF Corporation), and boscalid (Endura fungicide, $70 \% \mathrm{wt} / \mathrm{wt}$; BASF Corporation) were used to determine fungicide resistance profiles in B. cinerea isolates. A discriminatory dose for each fungicide was used to distinguish sensitive and resistant isolates in mycelial relative-growth assays, as previously described (FernándezOrtuño et al. 2014; Weber and Hahn 2011). Briefly, Czapek-Dox agar medium (CzA) with cyprodinil at $4 \mu \mathrm{g} / \mathrm{ml}, 1 \%$ malt extract agar (MEA) with fludioxonil at $0.5 \mu \mathrm{g} / \mathrm{ml}$, fenhexamid at $50 \mu \mathrm{g} / \mathrm{ml}$, iprodione at $10 \mu \mathrm{g} / \mathrm{ml}$, pyraclostrobin at $10 \mu \mathrm{g} / \mathrm{ml}$, thiophanate-methyl at $100 \mu \mathrm{g} / \mathrm{ml}$, and $1 \%$ yeast bacto acetate agar (YBA) with boscalid at $75 \mu \mathrm{g} / \mathrm{ml}$ were used to assess isolate in vitro sensitivity to cyprodinil, fludioxonil, iprodione, fenhexamid, pyraclostrobin, thiophanate-methyl, and boscalid, respectively. The addition of salicylhydroxamic acid at $100 \mu \mathrm{g} / \mathrm{ml}$ was included to suppress the alternative respiration pathway when assessing pyraclostrobin sensitivity. CzA medium was used to support the growth of isolates on nonamended medium. For all isolate sensitivity assessments, 24-well plates were used to determine mycelial relative growth, as described previously (Fernández-Ortuño et al. 2014).

Following sensitivity assessments, logistic regression and contingency tables were used to examine relationships between the cooccurrence of resistance to one fungicide class and resistance to another class, resistance to one fungicide class and the multiple resistance phenotype, and the multiple resistance phenotype and year of collection. For each isolate, the presence or absence of resistance to particular fungicide or a multifungicide-resistant phenotype was scored on a binary scale, with " 1 " denoting resistance or multifungicideresistant phenotype and " 0 " denoting sensitivity or resistance to a single fungicide. Logistic regression was preformed using the FREQ procedure of SAS 9.4 (SAS Institute) with Pearson $\chi^{2}$ statistics and the CMH option to calculate Cochran-Mantel-Haenszel statistics and provide logit estimates of the odds ratio and relative risks.

Influence of fungicide exposure on the development of multiple fungicide resistance. Assays using detached apple fruit were conducted to determine whether resistance to one or more classes of fungicides could be selected by a fungicide of a different class. Despite best efforts to surface sterilize strawberry fruit (data not shown), we were unable to keep the soft fruit from being infected and consumed by fungal contaminants. Hence, apple fruit were used instead of strawberry because of greater resiliency to fungal decay. The experiment was conducted with inoculum composed of a mix of isolates with different fungicide resistance phenotypes (see below). Selection for multiple fungicide resistance was assessed following exposure to a single fungicide class and in the absence of a fungicide selective pressure. For the fungicide selective-pressure treatment, the QoI fungicide pyraclostrobin was used.

Selection and characterization of fungal isolates for detachedfruit assays and their fungicide resistance phenotypes. In total, 11 single-spore isolates were used in this study: 5 with resistance to four chemical classes, including HA (fenhexamid), QoI (pyraclostrobin), MBC (thiophanate-methyl), and SDHI (boscalid) designated "resistant to four chemical classes" (4CCR); and six sensitive to all four fungicides, designated "OCCR". Isolates used in the assay were collected from strawberry fruit (10 isolates) or blackberry fruit (1 isolate) with BFR symptoms from different locations in North Carolina, South Carolina, Georgia, and Michigan (Supplementary Table S2). Prior to the assay, all isolates were stored at $4^{\circ} \mathrm{C}$ as dried mycelium on filter paper, as previously described (Hu et al. 2011). The sensitivity phenotype of the 11 isolates to cyprodinil, iprodione, fenhexamid, pyraclostrobin, thiophanate-methyl, and boscalid was determined visually on fungicide-amended 24 -well plates, as previously described (Fernández-Ortuño et al. 2014, 2015). 
Preparation of inoculum. To obtain conidia for inoculation of fruit, isolates were incubated on potato dextrose agar (PDA) at $22^{\circ} \mathrm{C}$ in darkness for 4 days, then placed under $24 \mathrm{~h}$ of fluorescent light at $22^{\circ} \mathrm{C}$ for 7 days to facilitate spore production. Each plate was flooded with $5 \mathrm{ml}$ of sterile deionized water, and the resulting conidial suspension was filtered through two layers of cheesecloth. The number of conidia in the suspension was estimated with the aid of a hemocytometer. The final concentration was adjusted to $5 \times 10^{5}$ conidia/ml. Conidia of isolates with the same fungicide sensitivity phenotype (0CCR and 4CCR) were mixed at various concentration to obtain multi-isolate suspensions of the 0CCR and 4CCR phenotypes. Specifically, suspensions of OCCR and 4CCR isolates were mixed 50/50\%, 95/5\%, and 99.5/0.5\%, respectively. Conidial suspensions of OCCR and 4CCR isolates were also tested separately to evaluate the sporulation of each suspension on apple fruit.

Inoculation of apple fruit. Organic 'Gala' apple fruit were washed with soap and water, surface sterilized for $5 \mathrm{~min}$ in $0.8 \%$ sodium hypochlorite solution, and rinsed three times with sterile distilled water. Organic fruit were chosen to avoid interference from field exposure to single-site fungicides, which are not allowed in organic production. When apple fruit had dried, they were cut into halves with a sterile scalpel, dipped for $30 \mathrm{~s}$ in a suspension containing Cabrio EG at $2.1 \mathrm{~g} /$ liter (representing label rate), and the halves were placed into plastic boxes ( 18 by 18 by $10 \mathrm{~cm}$ ) containing moist paper towels on the bottom to maintain high humidity. Plastic rings lined the bottom of the box to prevent movement of the apple halves. Nontreated fruit were dipped in distilled sterile water for $30 \mathrm{~s}$. Four hours later, a 30- $\mu$ l droplet of each mixed 0CCR/4CCR conidial suspension was placed on six equidistant points spread on each apple half. Control fruit received sterile water without conidia. Inoculated fruit halves were covered with lids and incubated for 7 days at room temperature. Subsequently, lids were removed and halves were incubated for another 7 days to facilitate sporulation. Spores were harvested from apple halves by scraping the sporulating area with premoistened sterile cotton swabs and transferred into $1.5-\mathrm{ml}$ Eppendorf tubes containing $1 \mathrm{ml}$ of sterile water. Harvested conidia collected from sporulating fruit from each treatment and each conidial mix were used to reinoculate apple halves treated with pyraclostrobin and left nontreated for a second and third round of selection. Six replicate apple halves were inoculated for each 0CCR/4CCR mixed conidial suspension and fungicide treatment with three consecutive rounds of selection. The entire experiment was repeated once in the form of a biological replicate.

Determination of frequency of OCCR and 4CCR conidia per transfer on apple halves. The frequency of conidia with resistance to pyraclostrobin was determined after each transfer and the frequency of conidia with resistance to the other three fungicides in the 4CCR phenotype (i.e., fenhexamid, thiophanate-methyl, and boscalid) was determined only after the last (third) transfer. A $100-\mu l$ aliquot of each conidial suspension originating from each apple half was spread onto MEA or YBA agar plates amended with a discriminatory dose of each fungicide, as outlined above. Three replicate plates were prepared for each conidial suspension. The plates were incubated for $16 \mathrm{~h}$ at $22^{\circ} \mathrm{C}$ to determine resistance to pyraclostrobin, fenhexamid, and thiophanate-methyl, and for $22 \mathrm{~h}$ at $22^{\circ} \mathrm{C}$ to determine resistance to boscalid. A spore-germination assay (Weber and Hahn 2011) was used to distinguish 4CCR and OCCR isolates. A minimum of 100 conidia per replicate plate was examined for germination microscopically to determine the frequency of 0CCR and 4CCR isolates. Single spores with 0CCR and $4 \mathrm{CCR}$ phenotypes were classified according to the following characteristics of germ-tube development on fungicide-amended media. Spores of 4CCR isolates developed full-length germ tubes on media amended with pyraclostrobin, fenhexamid, or boscalid, whereas spores of 0CCR isolates did not germinate. On the medium amended with thiophanate-methyl, spores of 4CCR isolates developed a full-size germ tube and germ tubes of OCCR isolates were tightly coiled, stunted, or highly branched.
Statistical analysis. All experiments to determine the effect of fungicide exposure on the development of multiple fungicide resistance were conducted twice. After repeating experiments, Bartlett's test for equal variances was performed to test whether the data on the sporulation of OCCR and 4CCR conidial suspensions and frequency of recovery for OCCR and 4CCR after apple inoculation from two independent experiments could be combined. The test indicated that variances of both sets of data between the experiments were equal $(P>0.05)$. Thus, all further analyses were conducted on merged data. Mean values of each OCCR and 4CCR phenotype for QoI-exposed and nonfungicide-exposed conditions were compared independently using the Student's $t$ test at $P=0.05$. The observed frequencies $(\%)$ of resistance were compared with the initial frequencies using a $\chi^{2}$ test. All percentage data were arcsinesquare-root transformed to stabilize the variances, and sporulation data were $\log _{10}$ transformed prior to analysis. All of the statistical analysis tests were performed using $\mathrm{R}$ software (version 3.2.0; R Foundation for Statistical Computing).

\section{RESULTS}

Frequency of resistance to single fungicide classes in blossom-derived Botrytis isolates. The frequency of isolates resistant to individual fungicide classes in strawberry fields from South Carolina and other states was investigated for all $B$. cinerea isolates. In South Carolina, the frequencies of isolates with resistance to pyraclostrobin, fenhexamid, boscalid, cyprodinil, iprodione, and fludioxonil increased from the 2011-12 season to 81.8, 31.5, 28.2, $25.5,8.2$, and $6.4 \%$ in the $2014-15$ season, respectively (Fig. 1A). The frequencies of isolates sensitive to all seven fungicides tested were $3.3,1.2,6.7$, and $3.3 \%$ in $2011-12,2012-13,2013-14$, and 2014-15, respectively (Fig. 1A). Resistance to thiophanate-methyl was consistently found at high frequencies, whereas resistance to fludioxonil was found only in a few isolates collected in the 2013-14

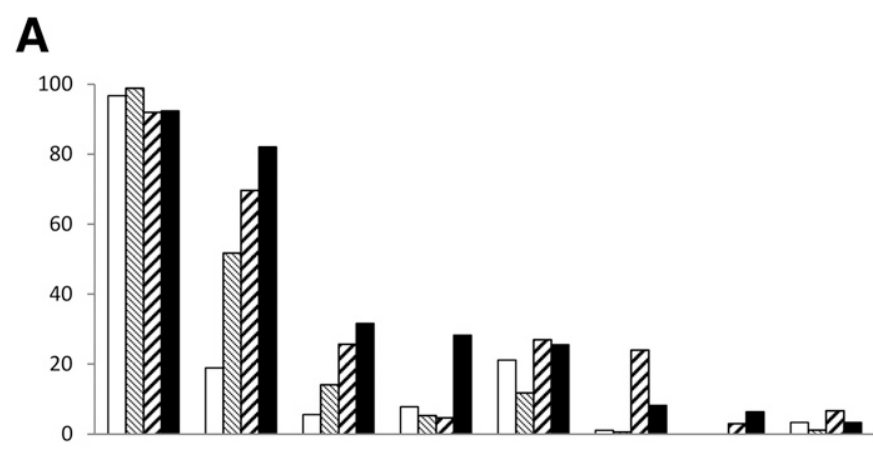

B

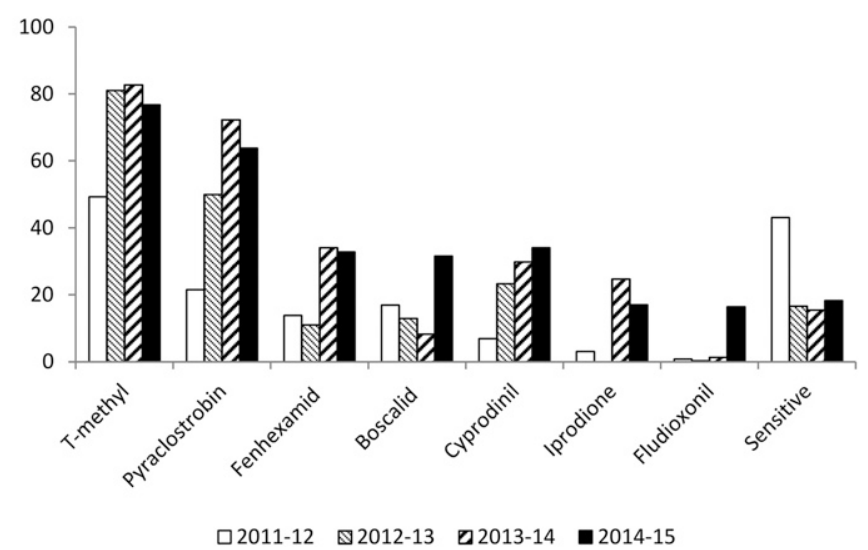

Fig. 1. Frequency (\%) of Botrytis cinerea isolates sensitive or resistant to seven chemical classes of fungicides collected from strawberry fields in $\mathbf{A}$, South Carolina and B, 10 other states. 
(3.0\%) and 2014-15 (6.4\%) seasons (Fig. 1A). Frequencies of isolates with resistance to pyraclostrobin, fenhexamid, or fludioxonil consistently increased from the 2011-12 to the 2014-15 seasons. Resistance to boscalid increased dramatically in season 2014-15 (28.2\%) compared with seasons 2011-12 (7.8\%), 2012-13 (5.3\%), and 2013-14 (4.7\%). Isolates collected in the 2014-15 season had the highest resistance frequencies for pyraclostrobin, fenhexamid, boscalid, and fludioxonil (Fig. 1A). Note that fludioxonil resistance was not associated with 7CCR in the first 2 years of monitoring.

Resistance profiles of isolates collected from the 10 other states revealed an increase of resistance to all chemical classes of fungicides used in this study from the 2011-12 season to the 2014-15 season (Fig. 1B). Similar to results obtained from South Carolina, resistance to thiophanate-methyl was found in nearly every location in all four seasons and resistance to fludioxonil was rarely found, with the exception of isolates collected in season 2014-15 (16.4\%). Isolates collected in the 2014-15 season showed the highest resistance frequencies for boscalid, cyprodinil, and fludioxonil (Fig. 1B).

Frequency of multifungicide-resistant isolates. In isolates from 2011-12 to 2014-15, there was a significant relationship between the presence of multifungicide-resistant isolates and season of collection $\left(\chi^{2}=183.7, P<0.0001\right)$. This is readily apparent in South Carolina isolates, as shown by the substantial decrease in the frequency of 1CCR isolates and a corresponding increase in the number of isolates with multifungicide-resistant phenotypes (2CCR, 3CCR, 4CCR, and so on) (Fig. 2A). In the 2011-12 season, 62.2, 21.1, 7.8, 4.4, and $1.1 \%$ of isolates were resistant to one (1CCR), two (2CCR), three (3CCR), four (4CCR), and five (5CCR) chemical classes of fungicides. The numbers of isolates in these multifungicide-resistant groupings changed over time, in that there was an increase of frequencies of isolates resistant to three and more chemical classes of fungicides (Fig. 2A). 6CCR isolates $(2.3 \%)$ were first detected in the 2013-14 season and 7CCR isolates $(0.3 \%$ ) were first detected in the $2014-15$ season (Fig. 2A). In the $2014-15$ season, $17.0,31.6,15.5,17.0,13.1,2.1$, and $0.3 \%$ of
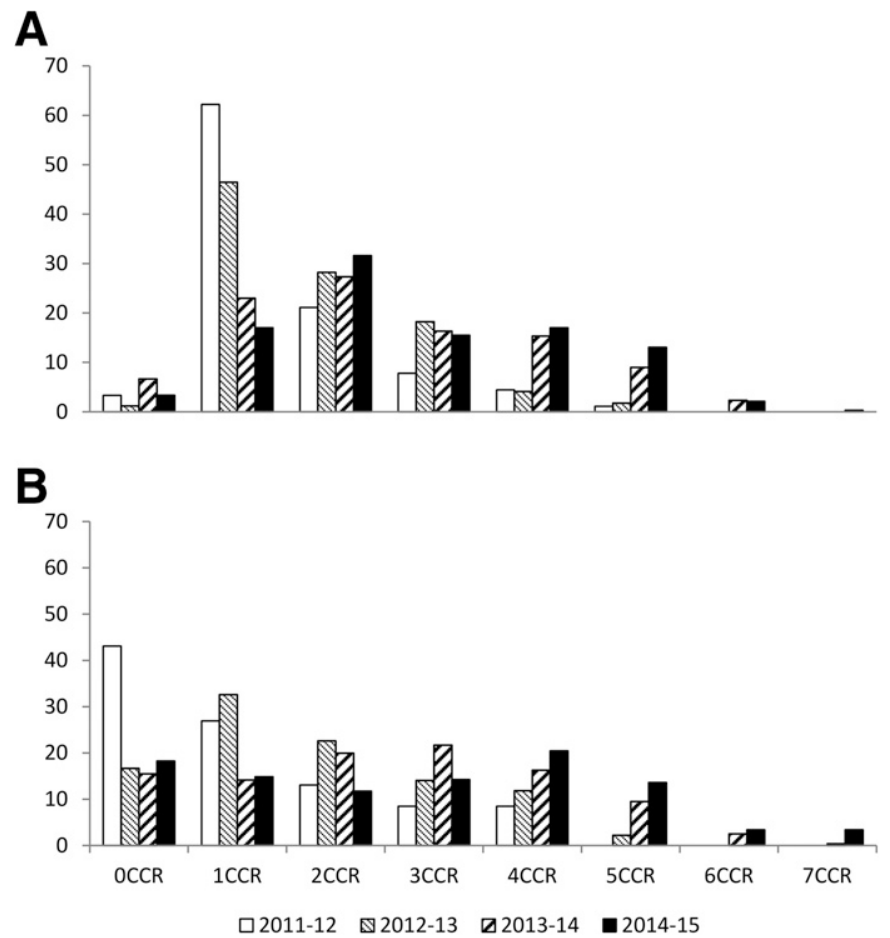

Fig. 2. Frequency (\%) of Botrytis cinerea isolates sensitive (0CCR) or resistant to one, two, three, four, five, six, or seven chemical classes of fungicides (1CCR to 7CCR) from strawberry fields in $\mathbf{A}$, South Carolina and $\mathbf{B}, 10$ other states. the isolates collected were $1 \mathrm{CCR}, 2 \mathrm{CCR}, 3 \mathrm{CCR}, 4 \mathrm{CCR}, 5 \mathrm{CCR}$, 6CCR, and 7CCR, respectively (Fig. 2A).

Across the 10 other states in the eastern United States, the majority of B. cinerea isolates collected in 2011-12 season were sensitive (0CCR) or 1CCR. In subsequent years, the frequency of isolates with resistance to more than one fungicide increased (Fig. 2B). Specifically, we observed an increase in 3CCR, 4CCR, 5CCR, 6CCR, and 7CCR isolate frequency. In addition, 6CCR and 7CCR isolates first emerged in season 2013-14 and increased in the following experimental season (2014-15) (Fig. 2B).

Occurrence and distribution of resistance to single fungicide classes in multifungicide-resistant isolates. Taking all 2,130 B. cinerea isolates together, $23.7,22.2,16.2,14.3,8.1$, and $1.8 \%$ had 1CCR, 2CCR, 3CCR, 4CCR, 5CCR, and 6CCR phenotypes, respectively. Compared with other fungicides, thiophanate-methyl resistance always occurred at the highest frequencies. Among 1CCR, 2CCR, 3CCR, 4CCR, 5CCR, and 6CCR isolates, the frequency of thiophanate-methyl resistant isolates ranged from $90.9 \%$ in $1 \mathrm{CCR}$ isolates to $100 \%$ in 5CCR and 6CCR isolates (Table 1). Isolates with resistance to thiophanate-methyl had 117.7 times greater odds of being multifungicide resistant than having resistance to a single fungicide $(95 \%$ confidence interval $[\mathrm{CI}]$ for logit estimated odds ratio 57.9 to $239.4, \chi^{2}=183.7, P<0.0001$ ). Pyraclostrobin resistance was the second most frequent phenotype in multifungicide-resistant isolates and occurred at high frequencies of $90.3 \%$ or higher in 2CCR to 6CCR isolates (Table 1). Isolates with resistance to pyraclostrobin had 462.9 times greater odds of being multifungicide resistant than having resistance to a single fungicide $\left(95 \% \mathrm{CI}\right.$ for logit estimated odds ratio 301.4 to $711.0, \chi^{2}=$ 1741.7, $P<0.0001)$. Interestingly, isolates with resistance to cyprodinil were often also associated with multifungicide resistance. The odds of an isolate with resistance to cyprodinil having multifungicide resistance was 126.9 times higher than an isolate having resistance to only a single fungicide (95\% CI for logit estimated odds ratio 47.2 to $340.9, \chi^{2}=398.7, P<0.0001$ ). Fludioxonil resistance was the least frequently observed, with only $4.37 \%$ (92 of 2,128) of the isolates having resistance to fludioxonil. For the 92 isolates in which resistance occurred, the odds of an isolate with resistance to fludioxonil having multifungicide resistance was 57.1 times higher than an isolate being resistant only to fludioxonil $(95 \% \mathrm{CI}$ for logit estimated odds ratio 7.9 to 410.2, $\left.\chi^{2}=53.0, P<0.0001\right)$.

Aside from the prevalence of resistance traits in multifungicideresistant isolates, the isolates with resistance to one fungicide often cooccurred with resistance to another fungicide. A pairwise comparison matrix of the frequency of resistance for each of the seven fungicides was conducted to examine co-occurrence of resistance between two classes of fungicides (Fig. 3). In this pairwise comparison, the highest frequency of co-occurring resistance was found between thiophanatemethyl and pyraclostrobin, in that isolates with resistance to thiophanate-methyl had 17.4 times greater odds of being resistant to pyraclostrobin (95\% CI for logit estimated odds ratio 12.4 to 24.5 ,

TABLE 1. Frequencies of multifungicide-resistant isolates and their phenotypes

\begin{tabular}{lcrrrrrr}
\hline & \multicolumn{7}{c}{ Resistance frequencies $(\%)^{\mathrm{a}}$} \\
\cline { 2 - 8 } Phenotype $(n)^{\mathrm{b}}$ & T-methyl & \multicolumn{1}{c}{ Pyr } & \multicolumn{1}{c}{ Cyp } & Fen & Bos & \multicolumn{1}{c}{ Ipr } & \multicolumn{1}{c}{ Flu } \\
\hline 1CCR (504) & 90.9 & 6.7 & 0.8 & 0.8 & 0.4 & 0.2 & 0.2 \\
2CCR (472) & 98.7 & 90.3 & 4.4 & 3.4 & 1.5 & 0.4 & 0.8 \\
3CCR (345) & 99.7 & 97.7 & 32.8 & 40.6 & 12.5 & 11.9 & 4.6 \\
4CCR (306) & 99.7 & 98.4 & 64.1 & 61.8 & 40.8 & 29.4 & 5.9 \\
5CCR (173) & 100 & 98.8 & 87.9 & 82.1 & 60.7 & 59.5 & 11.0 \\
6CCR (38) & 100 & 100 & 100 & 81.6 & 78.9 & 92.1 & 44.7 \\
\hline
\end{tabular}

a T-methyl $=$ thiophanate-methyl, $\mathrm{Pyr}=$ pyraclostrobin, $\mathrm{Cyp}=$ cyprodinil, Fen $=$ fenhexamid, Bos = boscalid, Ipr = iprodione, and Flu = fludioxonil.

${ }^{b}$ Isolates resistant to one, two, three, four, five, or six chemical classes of fungicides $=1 \mathrm{CCR}$ to $6 \mathrm{CCR}$, respectively. 
$\left.\chi^{2}=408.8, P<0.0001\right)$. Of the isolates with resistance to thiophanate-methyl, $71.19 \%$ were also resistant to pyraclostrobin.

Competitiveness of multifungicide-resistant isolates in conidial mixtures recovered from apple. In mixtures with spores from sensitive isolates, the 4CCR isolates all but vanished from the inoculum harvested after three transfers on nontreated apple halves (Table 2). The decline of 4CCR isolates was rapid and occurred mostly after the first transfer, leading to a dominance of the OCCR isolates (Fig. 4A). After the third transfer, the frequency of 4CCR isolates had decreased from an initial 50 to $6.8 \%$. When 4CCR spores made up a small fraction $(0.5$ and $5 \%)$ of the spore mixture at initial (first) inoculation, resistant spores were no longer detected by the third transfer (Table 2). When apple fruit were treated with pyraclostrobin, approximately $93 \%$ of the spores recovered had the 4CCR phenotype after the first transfer regardless of the initial inoculum frequency (Fig. 4B; Table 2). Considering that overall germination frequency was approximately $95 \%$ for both 4CCR and 0CCR phenotypes, the remaining $7 \%$ of spores may have been nongerminated spores of 4CCR isolates rather than spores from 0CCR isolates (data not shown). No significant difference was found in sporulation between 4CCR and OCCR isolates after each transfer $(P>0.05$; data not shown).

Frequency of resistance to fenhexamid, thiophanate-methyl, and boscalid in $4 \mathrm{CCR} / 0 \mathrm{CCR}$ isolate mixtures recovered from apple with pyraclostrobin selective pressure. The distribution of resistance components was investigated in spores recovered from the $0.5 \%$ 4CCR/0CCR mixture inoculated to apple fruit. Analysis of the initial inoculum containing $0.5 \%$ 4CCR spores revealed resistance frequencies to thiophanate-methyl, pyraclostrobin, fenhexamid, and boscalid of $0.2,0.4,0.3$, and $0.3 \%$, respectively. In the absence of pyraclostrobin selection pressure, isolates with resistance to thiophanate-methyl, pyraclostrobin, fenhexamid, or boscalid were not detected after a single passage through nontreated apple halves (data not shown). The frequencies of resistance to thiophanate-methyl, pyraclostrobin, fenhexamid, and boscalid in isolates collected after three consecutive passages through pyraclostrobintreated apple halves increased to 92.5, 93.1, 92.5, and 92.1\%, respectively.

\section{DISCUSSION}

Location-specific fungicide resistance monitoring was conducted for 213 strawberry production fields between the 2011-12 and 2014-15 seasons. Growers participating in the monitoring program implemented specific fungicide resistance management programs in response to monitoring data. Specifically, growers implemented a rotational program of fungicides when there was little or no evidence of resistance following monitoring. If there was a low level of resistance (no more than one resistant isolate per field) to any of the seven registered classes of fungicides considered in this study, the grower restricted applications of the fungicide in question to one application per season and added fungicides of different chemical classes into the rotation. If there was high level of resistance (two or more resistant isolates per field) to any of the seven classes of fungicides, the grower avoided applications of the fungicide in question altogether and added fungicides of different chemical classes into the rotation. Over the 4-year study, resistance to several fungicide classes increased in strawberry fields in South Carolina and other states throughout the eastern United States. The frequency of isolates from all states with resistance to pyraclostrobin, fenhexamid, and cyprodinil consistently increased over the 4 years of monitoring. Across all years, the majority (71.9\%) of isolates with resistance to thiophanate-methyl were also resistant to pyraclostrobin. Given that thiophanate-methyl and pyraclostrobin resistance occurred in most isolates, it stands to reason that these two fungicides should not be used for resistance management or BFR management, for that matter. In contrast, the frequency of fludioxonil-resistant isolates was fairly low ( $<5 \%$ across all years), and isolates having fludioxonil resistance and resistance to any of the other six fungicides was similarly low $(<5 \%)$. Hence, fludioxonil may be the best rotational partner for fungicide resistance management. However, the use of fludioxonil by strawberry growers is complicated by the fact that the fungicide is currently only available for preharvest applications in a premix formulation with cyprodinil, marketed as Switch WG (Syngenta Crop Protection).

In South Carolina, isolates were collected each season from the same fields at the same times (prebloom) and, therefore, it is reasonable to assume that the majority of the inoculum collected over the 4 years for this study was subsequently exposed to the fungicide resistance management programs implemented by the

TABLE 2. Change of frequency of isolates resistant to four chemical classes of fungicides (4CCR) coinoculated with sensitive isolates (0CCR) on apple fruit halves at different spore ratios ${ }^{\mathrm{a}}$

\begin{tabular}{lcc}
\hline & \multicolumn{2}{c}{$\begin{array}{c}\text { Frequency on apple halves } \\
\text { after three transfers }(\%)\end{array}$} \\
\cline { 2 - 3 } $\begin{array}{l}\text { Frequency at initial } \\
\text { inoculation }(\%)\end{array}$ & Pyraclostrobin-treated & Nontreated \\
\hline 50 & $92.8^{*}$ & $6.8^{*}$ \\
5 & $92.1^{*}$ & 0 \\
0.5 & $92.5^{*}$ & 0
\end{tabular}

a Frequency of 4CCR spores after three transfers and at initial inoculation; * indicates a significant difference compared with the initial spore ratio in column 1 according to a $\chi^{2}$ test at $P=0.05$.

\begin{tabular}{|c|c|c|c|c|c|c|c|}
\hline & Thiophanate-methyl & Pyraclostrobin & Cyprodinil & Fenhexamid & Iprodione & Boscalid & Fludioxonil \\
\hline \multicolumn{8}{|c|}{ Thiophanate-methyl } \\
\hline \multicolumn{8}{|c|}{ Pyraclostrobin } \\
\hline \multicolumn{8}{|l|}{ Cyprodinil } \\
\hline \multicolumn{8}{|c|}{ Fenhexamid } \\
\hline \multicolumn{8}{|l|}{ Iprodione } \\
\hline Boscalid & & & & & & & \\
\hline Fludioxonil & & & & & & & \\
\hline
\end{tabular}

Fig. 3. Pairwise comparison matrix of the frequency of co-occurring resistance for each of the seven fungicides. Each pairwise comparison, represented by a shaded box, was using all 2,130 isolates from all seasons with simple logistic regression and contingency tables using the FREQ procedure of SAS 9.4 (SAS Institute). Boxes colored darkest gray indicate that more than $50 \%$ of the isolates in the pairwise combination had co-occurring resistance to the fungicides intersecting by row and column. Boxes in dark gray, light gray, and white indicate that 20 to $50 \%$ of isolates, 5 to $15 \%$ of isolates, and $<5 \%$ of the isolates, respectively, had co-occurring resistance to the fungicides intersecting by row and column. 
growers. The remaining postfruit production season population may then have provided the inoculum for the following season. It is also possible that at least some inoculum was already present on nursery stock in the form of latent infections. In other words, the prebloom inoculum in any given year may have originated from both the nursery and the producer farm. However, the nurseries supplying plants to growers in the region were using the same fungicide resistance management practices, because we provided our monitoring service to many of these nurseries as well (data not shown). Therefore, it is also reasonable to assume that at least some of the isolates coming in from nursery plants were exposed to fungicide selection pressures following the same fungicide resistance management practices.

Regardless of the source of inoculum, we were perplexed as to why the proposed resistance management strategies, that were designed to delay the onset of resistance, would increase the frequency of multifungicide-resistant isolates. Theoretically, a mechanism conferring simultaneous resistance to multiple fungicides could explain our observations. In this regard, drug efflux pump activity may come to mind. However, the discriminatory doses used in this study to distinguish sensitive from resistant isolates were set high enough for thiophanate-methyl, pyraclostrobin, fenhexamid, iprodione, and boscalid to allow only isolates with resistance mechanisms based on target gene mutations to survive (Fernández-Ortuño et al. 2014; Weber and Hahn 2011). Weber and Entrop (2011) provided preliminary evidence that an application of a fungicide in one class may lead to selection of resistance to a fungicide in an unrelated class in $B$. cinerea populations containing isolates with multifungicide resistance. For example, if $B$. cinerea isolates were resistant to thiophanate-methyl and boscalid, each application of boscalid or thiophanate-methyl would select for this dual-resistant phenotype. We hypothesize that such "selection by association" occurred in the present study. In a previous study, we established the existence of isolates that were resistant to up to five chemical classes of fungicides at low frequencies in many fields in South Carolina and North Carolina (Li et al. 2014a), and it is reasonable to assume that these resistant genotypes were already present at low frequencies in the strawberry fields we examined. In fact, we identified 5CCR isolates at low frequencies during the 2011-12 season (data not shown). Furthermore, we established the existence of 7CCR isolates on the east coast (Fernández-Ortuño et al. 2015). Hence, resistance management practices implemented by the growers may have selected for preexisting resistance to chemical classes that were not part of the selection pressure. In other words, fungicide resistance traits were selected that were only linked to the trait being selected rather than the selectable trait itself. For example, isolates with resistance to chemical classes $\mathrm{A}, \mathrm{B}$, and $\mathrm{C}$ would be selected by fungicides belonging to either of the three chemical classes. This may also explain the stable frequency of resistance to thiophanatemethyl despite the fact that this fungicide was no longer used in the strawberry production fields included in this study. Our previous studies showed that virtually all isolates with multifungicide resistance include resistance to thiophanate-methyl ( $\mathrm{Li}$ et al. 2014a) and, therefore, selection for thiophanate-methyl resistance may occur each time the population is exposed to a single-site-fungicide. Resistance to thiophanate-methyl is stable and has not been associated with reduced fitness in field isolates. Although these observations predominately applied to isolates from South Carolina strawberry fields, similar selections by association were made for isolates collected in other states as well.

Multifungicide resistance in $B$. cinerea field populations evolves through stepwise accumulation of resistance to single fungicide classes over years due to continuous selection and population diversification ( $\mathrm{Li}$ et al. 2014a). Selection for resistance to other, newly introduced fungicides was shown to have developed in populations already resistant to thiophanate-methyl, which was

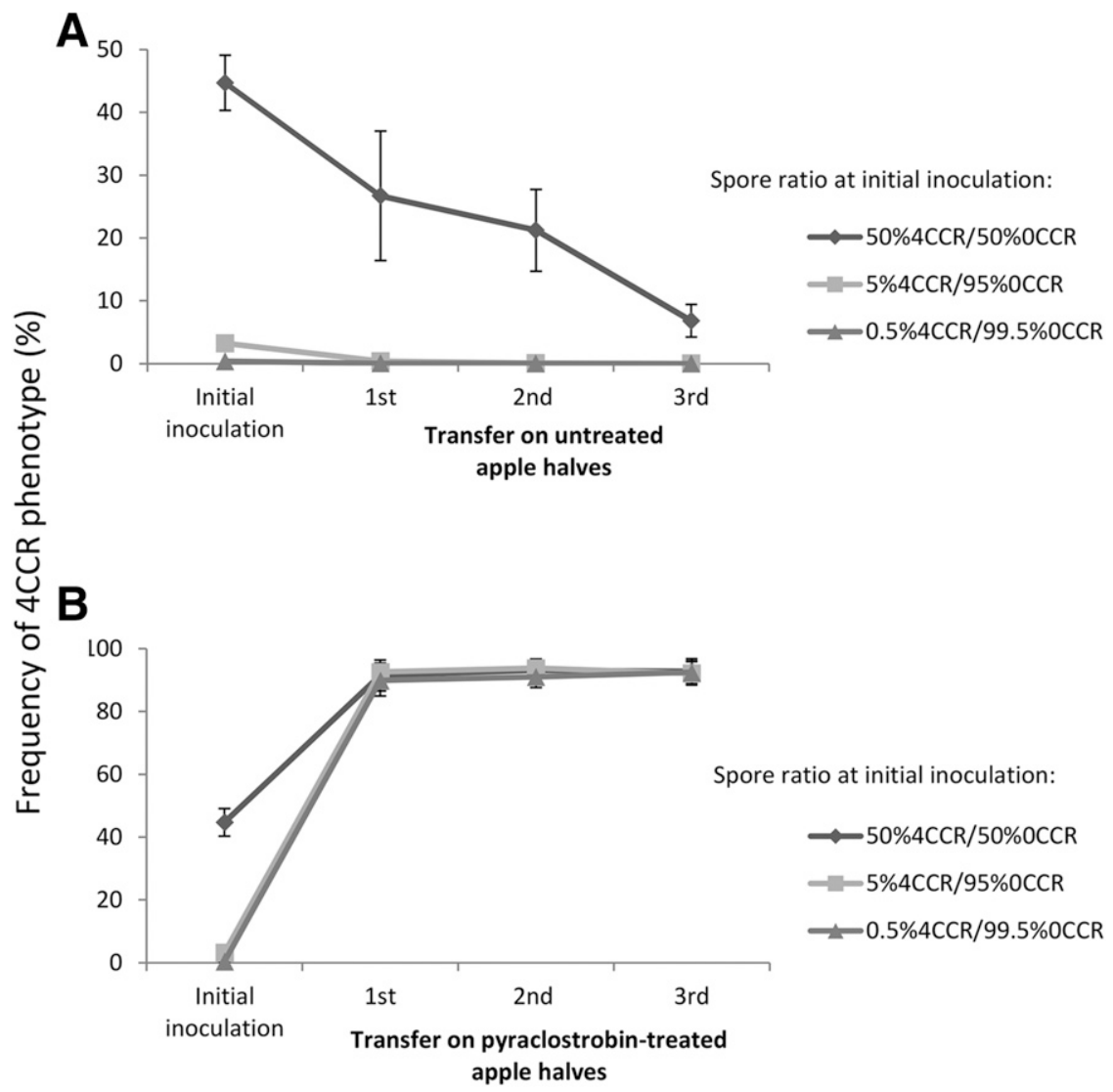

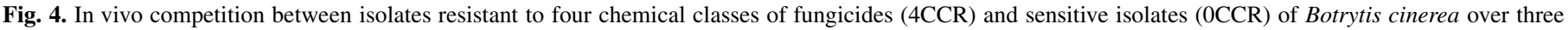
transfers on $\mathbf{A}$, nontreated and $\mathbf{B}$, pyraclostrobin-treated apple halves with varying spore ratios. 
introduced in the late 1970s for the control of many diseases of strawberry. Based on the proposed selection by association of multifungicide-resistant genotypes when multiple chemical classes are rotated, we would expect that the more chemical classes are involved in a resistance management program, the more selection pressure there is on isolates that are already resistant to all of these chemical classes of fungicides. Therefore, rotations and mixtures of single-site fungicides with different modes of action may help growers control disease in the short term but, in the long term, this strategy may not be sustainable if multifungicide resistance is already present in the population. The present 4-year study clearly showed an increase in resistance to fungicides in blossom-derived isolates from South Carolina strawberry fields and from fields in other states and, thus, supports our hypothesis. Our observation are consistent with a previous study that observed an increase in the frequency of $B$. cinerea isolates with resistance to one, two, or three chemical classes of fungicides from 2011-12 to 2012-13 (Fernández-Ortuño et al. 2014). An alternate explanation for our observations is that resistance to chemical classes of fungicides may not have already existed but, rather, accumulated in response of the selection pressure. This would have required a very short response time of 1 year or less between seasons for the emergence of new genotypes with the proper point mutation in target genes. Although this is possible, we believe that the more likely scenario is based on our hypothesis that the genotypes selected over the four experimental years had already existed.

The above-discussed concept of selection by association is based on the assumption that an isolate with resistance to, for example, chemical classes A, B, and C would be selected by all three chemical classes. That technically means that, for example, the application of chemical class A would also select for resistance to chemical classes B and C. This indirect selection of resistance is also referred to as genetic hitchhiking, a phenomenon that has been reported in the context of insecticide resistance (Yan et al. 1998). In the traditional sense, genetic hitchhiking is when an allele changes frequency not because it itself is under natural selection but because it is near another gene on the same chromosome that is undergoing a selective sweep (Lang et al. 2013). In our case, the role of driver and hitchhiker may be switched several times in a season. To our knowledge, selection by association in fungi has not been experimentally documented and, thus, proof of concept was established in this study. Apple fruit were used for the experiments because preliminary experiments with strawberry fruit, the original host of our isolates, resulted in contamination due to latent infections despite surface sterilization. A spore mixture of sensitive (0CCR) and 4CCR $B$. cinerea isolates was used to inoculate apple fruit treated with pyraclostrobin as a QoI selective pressure. As expected, isolates resistant to pyraclostrobin were selected and 0CCR isolates all but disappeared over three transfers. The surviving 4CCR isolates were resistant to pyraclostrobin as well as thiophanate-methyl, fenhexamid, and boscalid, although the latter three fungicides were never applied (data not shown). Thus, resistance to the latter three fungicides was selected not directly but by association, because the 4CCR isolates carried the genetic determinants for resistance to all four fungicides. Although it might be tempting to postulate the involvement of a multidrug efflux pump or other multiresistance mechanism, the molecular basis of simultaneous resistance in our 4CCR isolates to the four chemical classes of fungicides applied in this experiment was also based on mutations in four different target genes. They included G143A in the cytb gene, E198A in the $\beta$-tubulin, F412S or F412C in the erg 27 gene, and H272R or H272Y in the $s d h B$ gene (data not shown).

As mentioned above, our location-specific resistance profiling service and resulting resistance management program strongly influenced a grower's fungicide program. Fludioxonil and iprodione resistance was rarely found in the first two seasons and, thus, these compounds were often part of the recommendations for BFR management (data not shown). Fludioxonil is only sold in mixture with cyprodinil and resistance in fungal plant pathogens has been reported in only a few studies (Fernández-Ortuño et al. 2013a; Grabke et al. 2014; Leroch et al. 2013; Li and Xiao 2008; Li et al. 2014b; Zhao et al. 2010). Resistance frequencies to these three compounds increased over the course of the four seasons, which is consistent with our current understanding of direct selection for resistance.

As indicated above, the concept of selection by association would suggest that multifungicide-resistant isolates are selected each time a single-site fungicide is applied. Thus, we would have expected an even more rapid selection of 5CCR, 6CCR, and 7CCR fungicides, provided these genotypes were already present in the population and considering how rapidly B. cinerea can spread (Jarvis 1992). Although we are seeing an increase of isolates with simultaneous resistance to an increasing number of fungicides, 5CCR, 6CCR, and 7CCR isolates were still in the minority after the four experimental years. Why are we not seeing an even more rapid increase in multifungicide resistance? We believe that reduced fitness and competitive ability may help slow down this selection process during times when no fungicides are applied (many growers do not spray prior to bloom) or when fungicides are applied that do not select for resistance, such as captan or thiram. Reduced fitness of isolates with multifungicide resistance phenotypes has been described in B. cinerea isolates (Fernández-Ortuño et al. 2015; Veloukas et al. 2014). More importantly, 5CCR and, especially, 5CCR isolates containing the MDR1 phenotype did not successfully compete with sensitive isolates under controlled conditions (Chen et al. 2016). This supports the hypothesis that, during the process of accumulating single resistance over time ( $\mathrm{Li}$ et al. 2014a), fitness penalties are increased due to the implementation of an increasing number of disadvantageous genetic changes in the genome during selection pressure. Evidence for impaired competitive ability was provided in this study as well. In the absence of pyraclostrobin, the 4CCR isolates virtually disappeared and, thus, were outcompeted by 0CCR isolates after only three transfers. This observation is in agreement with previous findings showing that fitness of resistant isolates is impaired (Kim and Xiao 2011; Veloukas et al. 2014) and confirms that fitness cost reduces competitiveness in multifungicide-resistant isolates (Chen et al. 2016). Therefore, the advantage of being selected more favorably by the application of different single-site fungicides may be moderated by the inability to compete effectively with more fit genotypes in the field.

As mentioned above, fungal inoculum may be brought in with the transplants from nurseries. Recent studies have confirmed this hypothesis (Oliveira and Peres 2014; Oliveira et al. 2013) and our own research confirms that plants from nurseries harbored isolates resistant to multiple chemical classes of fungicides (Schnabel et al. 2015). Specifically, isolates from unsprayed strawberry plants of 14 of 15 fields in various East Coast states were already resistant to three and more chemical classes of fungicides (Schnabel et al. 2015). Additionally, we also have direct evidence that resistance is already present in nursery stock. A collection of 30 isolates obtained from berry fruit of a major planting stock provider was subjected to sensitivity assays and revealed the existence of multifungicideresistant phenotypes (data not shown). Therefore, it is possible that flower infections, at least in part, may have come from populations that were exposed to fungicide programs in nurseries. Resistance management in nurseries that produce strawberry tips and plugs for fruit producers must become an integral part of the overall plan to manage fungicide resistance.

In conclusion, our data suggest that current resistance management programs based on rotation and mixtures of different fungicide classes lead to increased frequencies of multifungicide resistance in $B$. cinerea populations of eastern strawberry fields. Unfortunately, as additional chemical classes are involved in rotation and mixtures, it seems that selection for isolates resistant to all single-site fungicides involved will happen more quickly despite fitness penalties via selection by association. Avoidance or reduction of selective fungicide 
applications may also be of benefit, in that a multifungicideresistant population may revert to sensitivity over time. Therefore, reducing applications of selective fungicides is one of the major goals in the southeastern United States and is implemented via decision support systems for growers (MacKenzie and Peres 2012; Pavan et al. 2011; Peres and Amiri 2013). Other means to reduce the number of applications of selective fungicides include the integration of biological control materials, emphasis on field sanitation, and use of clean planting stock.

\section{ACKNOWLEDGMENTS}

Technical Contribution Number 6392 of the Clemson University Experiment Station. This material is based upon work supported by the United States Department of Agriculture National Institute of Food and Agriculture (USDA-NIFA) under project number SC-1700501 and USDANIFA Specialty Crop Research Initiative grant number 2014-51181-22377.

\section{LITERATURE CITED}

Brent, K. J. 1988. Monitoring for fungicide resistance. In: Fungicide Resistance in North America. American Phytopathological Society, St. Paul, MN.

Brent, K. J., and Hollomon, D. W. 2007. Fungicide Resistance in Crop Pathogens: How Can It be Managed? 2nd ed. FRAC Monograph No. 2. Fungicide Resistance Action Committee, United Kingdom.

Bristow, P. R., McNicol, R. J., and Williamson, B. 1986. Infection of strawberry flowers by Botrytis cinerea and its relevance to grey mould development. Ann. Appl. Biol. 109:545-554.

Chen, S. N., Luo, C. X., Hu, M. J., and Schnabel, G. 2016. Fitness and competitive ability of Botrytis cinerea isolates with resistance to multiple chemical classes of fungicides. Phytopathology 106:997-1005.

Delp, C. J. 1980. Coping with resistance to plant disease control agents. Plant Dis. 64:652-657.

Fernández-Ortuño, D., Bryson, P. K., Grabke, A., and Schnabel, G. 2013a. First report of fludioxonil resistance in Botrytis cinerea from a strawberry field in Virginia. Plant Dis. 97:848.

Fernández-Ortuño, D., Chen, F., and Schnabel, G. 2012. Resistance to pyraclostrobin and boscalid in Botrytis cinerea isolates from strawberry fields in the Carolinas. Plant Dis. 96:1198-1203.

Fernández-Ortuño, D., Chen, F., and Schnabel, G. 2013b. Resistance to cyprodinil and lack of fludioxonil resistance in Botrytis cinerea isolates from strawberry in North and South Carolina. Plant Dis. 97:81-85.

Fernández-Ortuño, D., Grabke, A., Bryson, P. K., Amiri, A., Peres, N. A., and Schnabel, G. 2014. Fungicide resistance profiles in Botrytis cinerea from strawberry fields of seven southern U.S. states. Plant Dis. 98:825-833.

Fernández-Ortuño, D., Grabke, A., Li, X., and Schnabel, G. 2015. Independent emergence of resistance to seven chemical classes of fungicides in Botrytis cinerea. Phytopathology 105:424-432.

Fernández-Ortuño, D., and Schnabel, G. 2012. First report of thiophanatemethyl resistance in Botrytis cinerea on strawberry from South Carolina. Plant Dis. 96:1700.

Grabke, A., Fernández-Ortuño, D., Amiri, A., Li, X., Peres, N. A., Smith, P., and Schnabel, G. 2014. Characterization of iprodione resistance in Botrytis cinerea from strawberry and blackberry. Phytopathology 104:396-402.

Grabke, A., Fernández-Ortuño, D., and Schnabel, G. 2013. Fenhexamid resistance in Botrytis cinerea from strawberry fields in the Carolinas is associated with four target gene mutations. Plant Dis. 97:271-276.

Hu, M.-J., Cox, K. D., Schnabel, G., and Luo, C.-X. 2011. Monilinia Species causing brown rot of peach in China. PLoS One 6:e24990.

Jarvis, W. R. 1992. Managing Diseases in Greenhouse Crops. American Phytopathological Society, St. Paul, MN.
Kim, Y. K., and Xiao, C. L. 2011. Stability and fitness of pyraclostrobin- and boscalid-resistant phenotypes in field isolates of Botrytis cinerea from apple. Phytopathology 101:1385-1391.

Ktiller, W., and Scheinpflug, H. 1987. Fungal resistance to sterol biosynthesis inhibitors: A new challenge. Plant Dis. 71:1066-1074.

Lang, G. I., Rice, D. P., Hickman, M. J., Sodergren, E., Weinstock, G. M., Botstein, D., and Desai, M. M. 2013. Pervasive genetic hitchhiking and clonal interference in forty evolving yeast populations. Nature 500:571-574.

Leroch, M., Plesken, C., Weber, R. W. S., Kauff, F., Scalliet, G., and Hahn, M. 2013. Gray mold populations in German strawberry fields are resistant to multiple fungicides and dominated by a novel clade closely related to Botrytis cinerea. Appl. Environ. Microbiol. 79:159-167.

Li, H. X., and Xiao, C. L. 2008. Characterization of fludioxonil-resistant and pyrimethanil-resistant phenotypes of Penicillium expansum from apple. Phytopathology 98:427-435.

Li, X., Fernández-Ortuño, D., Chen, S., Grabke, A., Luo, C.-X., Bridges, W. C., and Schnabel, G. 2014a. Location-specific fungicide resistance profiles and evidence for stepwise accumulation of resistance in Botrytis cinerea. Plant Dis. 98:1066-1074.

Li, X., Fernández-Ortuño, D., Grabke, A., and Schnabel, G. 2014b. Resistance to fludioxonil in Botrytis cinerea isolates from blackberry and strawberry. Phytopathology 104:724-732.

MacKenzie, S. J., and Peres, N. A. 2012. Use of leaf wetness and temperature to time fungicide applications to control Botrytis fruit rot of strawberry in Florida. Plant Dis. 96:529-536.

Oliveira, M. S., Amiri, A., and Peres, N. A. 2013. The role of nursery plants as a potential source of inoculum for Botrytis cinerea and its impact on fungicide sensitivity. (Abstr.) Phytopathology 103:S2.107.

Oliveira, M. S., and Peres, N. A. 2014. Strawberry nursery plants as a source of Botrytis cinerea isolates resistant to fungicides. (Abstr.) Phytopathology 104:S3.86.

Pavan, W., Fraisse, C. W., and Peres, N. A. 2011. Development of a web-based disease forecasting system for strawberries. Comput. Electron. Agric. 75: 169-175.

Peres, N. A., and Amiri, A. 2013. Multi-year evaluation of standard- and model-timed fungicide applications for the control of Botrytis and anthracnose in commercial strawberry fields. (Abstr.) Phytopathology 103:S2.112.

Schnabel, G., Hu, M., and Fernández-Ortuño, D. 2015. Monitoring Resistance by Bioassay: Relating Results to Field Use Using Culturing Methods. Springer, Japan.

Staub, T. 1991. Fungicide resistance: Practical experience with antiresistance strategies and the role of integrated use. Annu. Rev. Phytopathol. 29: 421-442.

Staub, T., and Sozzi, D. 1984. Fungicide resistance: A continuing challenge. Plant Dis. 68:1026-1031.

van den Bosch, F., and Gilligan, C. A. 2008. Models of fungicide resistance dynamics. Annu. Rev. Phytopathol. 46:123-147.

Veloukas, T., Kalogeropoulou, P., Markoglou, A. N., and Karaoglanidis, G. S. 2014. Fitness and competitive ability of Botrytis cinerea field isolates with dual resistance to SDHI and QoI Fungicides, associated with several $s d h B$ and the $c y t b$ G143A mutations. Phytopathology 104:347-356.

Weber, R. W. S., and Entrop, A.-P. 2011. Multiple Fungicide Resistance in Botrytis: A Growing Problem in German Soft-Fruit Production. Intech Publishing, Rijeka, Croatia.

Weber, R. W. S., and Hahn, M. 2011. A rapid and simple method for determining fungicide resistance in Botrytis. J. Plant Dis. Prot. 118:17-25.

Yan, G., Chadee, D. D., and Severson, D. W. 1998. Evidence for genetic hitchhiking effect associated with insecticide resistance in Aedes aegypti. Genetics 148:793-800.

Zhao, H., Kim, Y. K., Huang, L., and Xiao, C. L. 2010. Resistance to thiabendazole and baseline sensitivity to fludioxonil and pyrimethanil in Botrytis cinerea populations from apple and pear in Washington State. Postharvest Biol. Technol. 56:12-18. 